\title{
Subdoses de glyphosate não reduzem a produtividade da cultura do milho ${ }^{1}$
}

\author{
Glyphosate sub lethal rates do not reduce corn yield
}

\author{
Patrícia Aparecida de Carvalho Felisberto ${ }^{2}$; Paulo César Timossi ${ }^{3}$; Guilherme Felisberto ${ }^{4}$; \\ Andréia Rodrigues Ramos ${ }^{5}$
}

\begin{abstract}
Resumo - Na aplicação de herbicidas, utilizados como maturadores, deve ser levado em consideração as culturas cultivadas em áreas adjacentes, devido ao risco de ocorrência de deriva desses produtos. Partindo do pressuposto da sensibilidade da cultura do milho ao glyphosate, objetivou-se avaliar o efeito de subdoses de glyphosate na cultura do milho quando aplicadas em estádios de desenvolvimento V4 e V8. O experimento foi instalado em Jataí, GO, constituído de 10 tratamentos estabelecidos no delineamento com blocos casualizados em esquema fatorial $2 \times 5$, com quatro repetições. O primeiro fator correspondeu à aplicação do maturador nos estádios V4 e V8 de desenvolvimento da cultura do milho e o segundo fator às subdoses $(0 ; 3 ; 6 ; 9$ e $12 \%$ de 216,0 $\mathrm{g} \mathrm{ha}^{-1}$ e.a.) de glyphosate. Cada parcela experimental continha 10 linhas do híbrido SYN 7G17 com 9 metros de comprimento. Efeitos de fitotoxicidade visual nas plantas não foram perceptíveis em ambos estádios de aplicação aos 7, 14, 21, 28 e 42 dias após aplicação. Componentes de crescimento e de produção não foram afetados por subdoses de até $12 \%(25,92 \mathrm{~g}$ ha ${ }^{-1}$ e.a.) de glyphosate.
\end{abstract}

Palavras-chaves: deriva simulada de herbicidas; inibidor da EPSPs; fitotoxicidade; Zea mays

\begin{abstract}
In herbicides spraying, used as ripeners, should be considered the presence of crops growing in adjacent areas, due to the risk of drift of these products. In this context, assuming the sensitivity of corn to this ripener, the aim of this research was to evaluate the effect of glyphosate sublethal rates in corn when applied to V4 and V8 stages of development. The experiment was conducted in Jataí, GO, consisted of 10 treatments established in randomized blocks design where the treatments were arranged in factorial $2 \times 5$, with four replications. First factor corresponded to spraying at the V4 and V8 stages of development of the corn and the second factor to sublethal rates $\left(0 ; 3 ; 6 ; 9\right.$ and $12 \%$ of $216.0 \mathrm{~g} \mathrm{ha}^{-1}$ a.e.) of glyphosate. Each plot contained 10 rows of hybrid SYN 7G17 with 9 meters length. Visual phytotoxicity effects on plants were not noticeable in both stages of application at 7, 14,21, 28 and 42 days after application. Growth and production components were not affected by sublethal rates up to $12 \%$ ( $25.92 \mathrm{~g} \mathrm{ha}^{-1}$ a.e.) of glyphosate.

Keywords: herbicides simulated drift; EPSPs inhibitor; phytotoxicity; Zea mays

\footnotetext{
${ }^{1}$ Recebido para publicação em 27/07/2016 e aceito em 17/08/2016.

2 Doutoranda em Agronomia, Universidade Estadual Paulista "Júlio de Mesquita Filho" (UNESP) Faculdade de Ciências Agrárias e Veterinárias, Câmpus de Jaboticabal, Via de acesso prof. Donato Castellane, s/n, CEP 14884-900, Jaboticabal, SP. E-mail: <pa_carvalho@ymail.com>.

3 Docente do Curso de Agronomia, Universidade Federal de Goiás (UFG), Regional Jataí. E-mail: <ptimossi2004@yahoo.com.br>.

${ }^{4}$ Doutorando em Agronomia, Universidade Estadual Paulista "Júlio de Mesquita Filho" (UNESP) Faculdade de Ciências Agrárias e Veterinárias, Câmpus de Jaboticabal. E-mail: 〈gfelisberto@outlook.com>.

${ }^{5}$ Doutoranda em Agronomia, Universidade Estadual Paulista "Júlio de Mesquita Filho" (UNESP) Faculdade de Ciências Agrárias, Câmpus de Botucatu. E-mail: <andreia-agro@ @otmail.com>.
} 
O fornecimento de matéria-prima de qualidade tecnológica ao longo do ano e que garanta retorno econômico é uma das maiores necessidades da indústria sucroalcooleira (Caputo et al. 2008; Leite et al., 2011). Com a finalidade de facilitar o planejamento de colheita e aumentar a produtividade da cana-deaçúcar, tem-se utilizado maturadores químicos, tais como ethephon, trinexapac ethyl, sulfometuron methyl e glyphosate (Dalley e Richard Júnior, 2010; Leite et al., 2011; Felisberto et al., 2015; Viana et al., 2015). A aplicação desses produtos ocorre por meio de pulverização aérea, o que aumenta as chances de ocorrer deriva em outras culturas circunvizinhas, como o milho, se as condições meteorológicas não forem adequadas (Felisberto et al., 2015).

$\mathrm{Na}$ aplicação aérea, a pulverização deve atingir o seu alvo sem que ocorra deriva, garantindo a segurança das culturas vizinhas. Segundo Tuffi Santos et al. (2007), os danos causados pela deriva dependem da concentração e quantidade do princípio ativo que chega às culturas. Ainda, o risco de ocorrência de deriva nas aplicações está relacionado com o diâmetro de gotas, com a velocidade de deslocamento horizontal (Cunha, 2008), e condições climáticas como vento, temperatura e umidade relativa do ar.

O glyphosate, herbicida não-seletivo de ação sistêmica do grupo químico glicina substituída, é um dos maturadores utilizados em cultivos de cana-de-açúcar. Essa molécula inibe a enzima 5-enolpiruvilshikimato-3-fosfato sintase (EPSPs), a qual está presente na rota de síntese dos aminoácidos aromáticos fenilalanina, tirosina e triptofano e metabólitos secundários (Means e Kremer, 2007). O bloqueio dessa rota resulta no acúmulo de chiquimato nos tecidos das plantas levando à deficiência significante de lignina, alcaloide e flavonoides, diminuição da fixação de $\mathrm{CO}_{2}$ e produção de biomassa (Olesen e Cedergreen, 2010).

$\mathrm{Na}$ literatura existem diversos trabalhos com glyphosate, tais como maturador (Silva et al., 2010), efeito "hormesis" (Duke et al., 2006; Cedergreen, 2008; Velini et al., 2008; Velini et al., 2010; Belz e Duke, 2014), seletividade (Anzalone et al., 2014; Schrübbers et al., 2014), deriva simulada em cafeeiro (França et al., 2013; Carvalho et al., 2014) e doses reduzidas em milho (Magalhães et al., 2001). No entanto, pesquisas visando avaliar a fitotoxicidade na cultura do milho em campo, provenientes da aplicação de subdoses do maturador de cana-deaçúcar glyphosate em diferentes estádios de desenvolvimento, ainda não foram relatadas.

A cultura do milho, assim como em outras culturas circunvizinhas da cana-deaçúcar (Correia e Leite, 2012; Correia e Vilella, 2015), está sujeita ao risco de deriva ou erros de aplicação de maturadores (Felisberto et al. 2015). Neste contexto, buscando responder as indagações de pesquisadores, técnicos e produtores de cana-de-açúcar e milho, e partindo da hipótese de sensibilidade da cultura do milho ao maturador de cana-de-açúcar glyphosate, objetivou-se avaliar o efeito de subdoses desse maturador na cultura do milho quando aplicadas em estádios de desenvolvimento V4 e V8.

$\mathrm{O}$ experimento foi conduzido no município de Jataí, GO, sob coordenadas geográficas de $17^{\circ} 55^{\prime} 37,3^{\prime}, \mathrm{S}, 51^{\circ} 43^{\prime} 4,7^{\prime}$ ' $\mathrm{O}$ e altitude de $663 \mathrm{~m}$. O clima segundo Köppen (1931) é classificado como Awa, tropical de savana, mesotérmico, com duas estações bem definidas, com verão chuvoso e inverno seco, o que propicia duas safras anuais de alto rendimento em sistema de rotação soja-milho. Segundo INMET (2014), a temperatura média máxima e mínima da região, no período de janeiro a junho do ano de 2014 , foi de $23,4^{\circ} \mathrm{C}$ e $21,8^{\circ} \mathrm{C}$, respectivamente, com total de precipitação pluvial nesse período de aproximadamente 796 mm (Figura 1).

$\mathrm{O}$ solo da área experimental é classificado como Latossolo Vermelho distroférrico de textura argilosa. Uma amostra composta do solo da área experimental foi enviada para análise química para fins de 
fertilidade e determinação da correção do solo e adubação (Tabela 1).

O experimento foi constituído de 10 tratamentos estabelecidos em delineamento com blocos casualizados em arranjo fatorial $2 \times 5$, com quatro repetições. $\mathrm{O}$ primeiro fator correspondeu à aplicação nos estádios V4 e V8 de desenvolvimento da cultura do milho e o segundo fator às subdoses de $0 ; 6,48 ; 12,96$; 19,44 e 25,92 g ha-1 e.a. de glyphosate (Roundup Original, $360 \mathrm{~g} \mathrm{ha}^{-1}$ e.a., CS, Monsanto), o que corresponde a $0 ; 3 ; 6 ; 9$ e $12 \%$ da dose recomendada pelo fabricante de glyphosate como maturador de cana-de-açúcar (216,0 $\mathrm{g} \mathrm{ha}^{-1}$ e.a.).

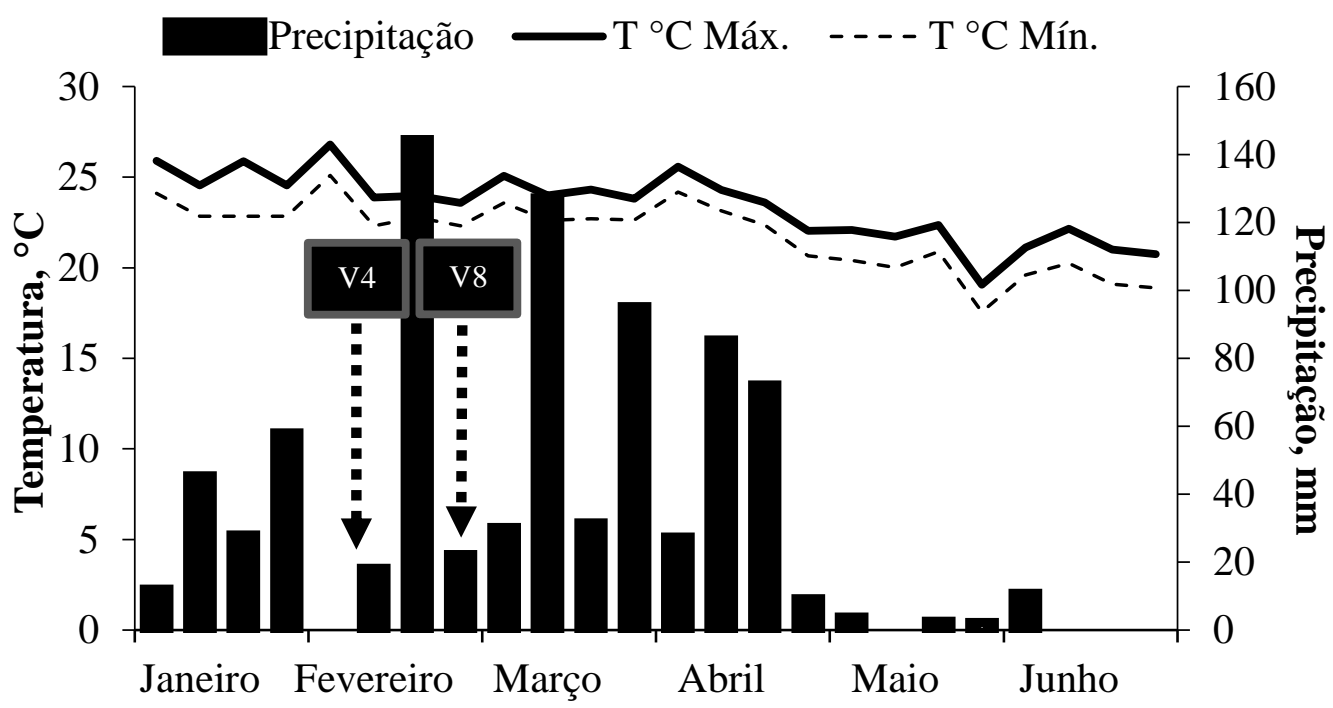

Figura 1. Dados climatológicos e estádios fenológicos V4 e V8 da cultura do milho que receberam aplicação de subdoses de glyphosate, no período de janeiro a junho de 2014. Jataí (GO), 2014.

Tabela 1. Análise química do solo da camada de 0 a 20 cm da área experimental. Jataí (GO), 2014.

\begin{tabular}{ccccccccccc}
\hline $\mathrm{pH}$ & $\mathrm{M} . \mathrm{O}$. & $\mathrm{P}$ & $\mathrm{H}+\mathrm{Al}$ & $\mathrm{K}$ & $\mathrm{Ca}$ & $\mathrm{Mg}$ & $\mathrm{SB}$ & $\mathrm{CTC}$ & $\mathrm{V}$ & ${\mathrm{S}-\mathrm{SO}_{4}{ }^{2-}}^{-{ }^{-3}}$ \\
\hline $\mathrm{CaCl}_{2}$ & $\mathrm{~g} \mathrm{dm}^{-3}$ & $\mathrm{mg} \mathrm{dm}^{-3}$ & $------------------\mathrm{cmol}_{\mathrm{c}} \mathrm{dm}^{-3}------------------$ & & $\%$ & $\mathrm{mg} \mathrm{dm}^{-3}$ \\
\hline 4,7 & 40 & 4,82 & 4,54 & 0,12 & 1,75 & 0,31 & 2,18 & 6,72 & 32,4 & 8,6 \\
\hline
\end{tabular}

$\mathrm{P}, \mathrm{K}$ - Mehlich-1; Ca, Mg - KCl.

A semeadura do milho (Híbrido SYN 7G17) foi realizada em 22/01/2014 com adubação de $500 \mathrm{~kg} \mathrm{ha}^{-1}$ 08-20-18 + 0,3\% Zn. Cada unidade experimental foi constituída por 10 linhas espaçadas a 0,45 metros e comprimento de 9 metros. A população estimada foi de 66.667 plantas ha ${ }^{-1}$. Aos 9 dias após a semeadura (DAS) realizou-se aplicação dos herbicidas $2.000 \mathrm{~g} \mathrm{ha}^{-1}$ i.a. de atrazine (Atrazina Atanor $50 \mathrm{SC}, 500 \mathrm{~g} \mathrm{~L}^{-1}$ i.a., SC, Atanor) e $100,8 \mathrm{~g} \mathrm{ha}^{-1}$ i.a. de tembotrione (Soberan, $420 \mathrm{~g} \mathrm{~L}^{-1}$ i.a., SC, Bayer) para manejo de plantas daninhas em área total. A adubação de cobertura foi realizada aos 24 DAS com 250 $\mathrm{kg} \mathrm{ha}^{-1}$ de ureia.

Aos 20 e 36 DAS, quando as plantas de milho se encontravam com 4 (V4) e 8 (V8) folhas totalmente expandidas, foram realizadas as aplicações das subdoses de glyphosate em 6 linhas centrais da parcela deixando-se duas linhas de cada lado sem aplicação para serem utilizadas como testemunha lateral das avaliações visuais. Para a aplicação utilizou-se um pulverizador costal pressurizado à $\mathrm{CO}_{2}$, munido de barra com 6 pontas de jato plano DG 11002, espaçados de $0,50 \mathrm{~m}$, com pressão constante de $280 \mathrm{kPa}$ e volume de calda 
equivalente a $200 \mathrm{~L} \mathrm{ha}^{-1}$. Uma lona de plástico foi posicionada ao lado da aplicação para proteger as outras parcelas e as testemunhas laterais. Na aplicação aos 20 DAS, as condições climáticas no momento da aplicação (11h08min) foram de $28,8^{\circ} \mathrm{C}$ de temperatura, $71 \%$ de umidade relativa do ar e $8,4 \mathrm{~km} \mathrm{~h}^{-1}$ de velocidade do vento. Na aplicação aos $36 \mathrm{DAS}$, as condições climáticas durante a aplicação $(09 \mathrm{~h} 26 \mathrm{~min})$ foram de $33,9^{\circ} \mathrm{C}$ de temperatura, $50,1 \%$ de umidade relativa do ar e ventos de 3,2 $\mathrm{km} \mathrm{h}^{-1}$.

Foram realizadas avaliações visuais dos efeitos de fitotoxicidade com base em escala de notas de intoxicação de plantas do EWRC (1964), que varia de 1 a 9, onde o menor valor representa ausência de fitotoxicidade e o maior valor, o caso mais extremo, ou seja, morte das plantas. Em relação ao desenvolvimento das plantas, atribuiu-se notas de porcentagem visual (0 a 100\%) de retardo no desenvolvimento aos $7,14,21,28$ e 42 dias após a aplicação (DAA) tomando-se como referência a testemunha lateral.

No estádio de desenvolvimento R1 (florescimento) foram determinados o diâmetro do colmo (DC), altura de inserção da primeira espiga (AIE) e altura de planta (AP). Para a determinação do DC foi utilizado paquímetro digital graduado em milímetros com medição no segundo internódio a partir da superfície do solo. A AIE e AP foram determinadas com uma régua topográfica graduada em centímetros, sendo que para a obtenção da AIE considerouse a distância entre a superfície do solo até a inserção da primeira espiga e para AP considerou-se a distância entre a superfície do solo e o ponto de inserção da folha bandeira. Todas as avaliações foram realizadas aleatoriamente em 10 plantas de cada parcela.

Em cada unidade experimental colheuse três linhas centrais de milho com quatro metros de comprimento (área útil), totalizando $5,4 \mathrm{~m}^{2}$ de área colhida. Separou-se aleatoriamente 10 espigas para determinar o número de fileiras de grãos $(\mathrm{FG})$, número de grãos por fileira (GF) e comprimento de espiga (CE). O FG e o GF foram obtidos a partir de simples contagem. $\mathrm{O} \mathrm{CE}$ foi determinado com o auxílio de fita métrica a partir da base da espiga até onde continha grão. O tamanho de grão (TG) foi calculado a partir da subtração do valor encontrado entre diâmetro da espiga e diâmetro de sabugo e divisão do resultado por dois.

A produtividade (PROD) foi obtida a partir da massa dos grãos contidos na área útil de cada parcela, mediante pesagem (expressa em $\mathrm{kg} \mathrm{ha}^{-1}$ ) e ajustada para $13 \%$ de teor de água. Para determinação da massa de 1000 grãos (M1000), foram realizadas contagens ao acaso de oito repetições de 100 grãos (Brasil, 2009), que tiveram suas massas determinadas e ajustadas para $13 \%$ de teor de água, possibilitando estimar através da média, a massa de 1000 grãos. Os dados obtidos foram submetidos à análise de variância por meio do teste $\mathrm{F}$ a $5 \%$ de significância.

Dentre as variáveis estudadas, não foi observado interação significativa entre os fatores (aplicação nos estádios de desenvolvimento V4 e V8 e subdoses de glyphosate). Também não foi encontrado diferenças significativas quando esses fatores foram analisados isoladamente. Portanto, a aplicação de glyphosate nos estádios V4 e V8 de desenvolvimento da cultura do milho (Tabela 2) e as subdoses de glyphosate (Tabela 3), não influenciaram nos valores de TG, FG, GF, DC, AIE, EST, M1000 e PROD.

Da mesma forma, as plantas de milho não apresentaram desenvolvimento afetado, ou seja, sem retardo visual no desenvolvimento das plantas aos 7, 14, 21, 28 e 42 dias após a aplicação (DAA) e as notas de EWRC foram iguais a 1 (sem dano visual de fitotoxicidade) para todas as avaliações. Magalhães et al. (2001) também não encontraram reduções na produtividade da cultura do milho em experimento de simulação de deriva de glyphosate em doses de 2 a $4 \%$ da dose recomendada como herbicida (1440 $\mathrm{g} \mathrm{ha}^{-1}$ e.a.) aplicadas em V6. 
Tabela 2. Médias de tamanho de grão (TG), número de fileiras de grãos por espiga (FG), número de grãos por fileira (GF), diâmetro do colmo (DC), altura de inserção da primeira espiga (AIE), altura de plantas (AP), número de plantas $\mathrm{m}^{-2}$ (EST), massa de 1000 grãos (M1000) e produtividade (PROD), analisados em relação à aplicação de subdoses de glyphosate nos estádios de desenvolvimento (E) da cultura do milho V4 e V8. Jataí (GO), 2014.

\begin{tabular}{cccccccccc}
\hline \multirow{2}{*}{ E } & TG & FG & GF & DC & AIE & AP & EST & M1000 & PROD \\
\cline { 2 - 9 } & -- mm -- & -- unidades -- & mm & $-----\mathrm{m}----$ & $\mathrm{pl} \mathrm{m}^{-2}$ & $\mathrm{~g}$ & $\mathrm{~kg} \mathrm{ha}^{-1}$ \\
\hline V4 & 10,38 & 15,69 & 30,67 & 25,44 & 1,36 & 2,33 & 7,55 & 285,13 & 7971,97 \\
V8 & 10,20 & 15,52 & 29,55 & 25,38 & 1,36 & 2,32 & 7,46 & 279,81 & 7825,08 \\
\hline F & $1,73^{\mathrm{ns}}$ & $0,95^{\mathrm{ns}}$ & $3,91^{\mathrm{ns}}$ & $0,02^{\mathrm{ns}}$ & $0,01^{\mathrm{ns}}$ & $0,15^{\mathrm{ns}}$ & $0,41^{\mathrm{ns}}$ & $1,65^{\mathrm{ns}}$ & $0,41^{\mathrm{ns}}$ \\
$\mathrm{CV}(\%)$ & 4,32 & 3,55 & 5,95 & 5,44 & 5,69 & 3,56 & 5,51 & 4,64 & 9,22 \\
\hline
\end{tabular}

ns, não significativo pelo Teste $\mathrm{F}$ a $\mathrm{p}<0,05 ; \mathrm{CV}$, coeficiente de variação; $\mathrm{pl} \mathrm{m}^{-2}$, plantas $\mathrm{m}^{-2}$.

Em outro estudo com simulação de deriva de glyphosate na cultura do sorgo, AlKhatib et al. (2003) observaram sintomas como clorose internervuras e nanismo da planta em doses de 71 e $168 \mathrm{~g} \mathrm{ha}^{-1}$ e.a. de glyphosate. Em doses maiores (404 e $1121 \mathrm{~g} \mathrm{ha}^{-1}$ e.a. de glyphosate) esses mesmos autores verificaram nanismo grave, clorose internerval e enrugamento das folhas, aos 6 DAA.

Pode-se afirmar que no presente estudo, sintomas visuais de fitotoxicidade e redução de produtividade não foram encontrados devido às baixas doses utilizadas. Segundo Reddy et al. (2008) o glyphosate, de maneira similar ao que ocorre no solo, pode ser metabolizado em ácido aminometilfosfônico (AMPA), tendo sua atividade reduzida. As baixas doses utilizadas somada a menor atividade do AMPA quando comparado ao glyphosate podem ser as causas da ausência da fitotoxicidade. Portanto, a cultura do milho mostrou-se seletiva para subdoses de até 25,92 $\mathrm{g} \mathrm{ha}^{-1}$ e.a. de glyphosate.

Velini et al. (2008) observaram efeito "hormesis" em plantas de milho em estádio V2V3 de desenvolvimento, mediante a aplicação de subdoses de glyphosate, diferentemente do observado no presente estudo. A ausência de efeito hormético pode ser explicada pela considerável influência de fatores como clima, cultivar, estádio de desenvolvimento da planta e a formulação do glyphosate utilizada (Velini et al., 2010). Assim, resultados diferentes poderiam ser observados se a aplicação das subdoses de glyphosate fosse realizada com cultivares, formulações e em estádios de desenvolvimento diferentes dos estudados neste trabalho.

Tabela 3. Médias de tamanho de grão (TG), número de fileiras de grãos por espiga (FG), número de grãos por fileira (GF), diâmetro do colmo (DC), altura de inserção da primeira espiga (AIE), altura de plantas (AP), número de plantas $\mathrm{m}^{-2}$ (EST), massa de 1000 grãos (M1000) e produtividade (PROD), analisados em relação às subdoses de glyphosate (SG). Jataí (GO), 2014.

\begin{tabular}{|c|c|c|c|c|c|c|c|c|c|}
\hline \multirow{2}{*}{ SG } & $\mathrm{TG}$ & FG & GF & $\mathrm{DC}$ & AIE & $\mathrm{AP}$ & \multirow{2}{*}{$\begin{array}{l}\mathrm{EST} \\
\mathrm{pl} \mathrm{m}^{-2} \\
\end{array}$} & \multirow{2}{*}{$\frac{\mathrm{M} 1000}{\mathrm{~g}}$} & \multirow{2}{*}{$\begin{array}{l}\text { PROD } \\
\mathrm{kg} \mathrm{ha}^{-1} \\
\end{array}$} \\
\hline & $--\mathrm{mm} \mathrm{--}$ & \multicolumn{2}{|c|}{-- unidades -- } & $\mathrm{mm}$ & \multicolumn{2}{|c|}{----- m ----- } & & & \\
\hline 0,00 & 10,77 & 15,98 & 31,05 & 26,14 & 1,43 & 2,37 & 7,27 & 285,50 & 7884,09 \\
\hline 6,48 & 10,04 & 15,40 & 30,20 & 24,62 & 1,33 & 2,31 & 7,66 & 271,87 & 7709,91 \\
\hline 12,96 & 10,25 & 15,34 & 28,84 & 25,49 & 1,33 & 2,29 & 7,55 & 288,64 & 8105,84 \\
\hline 19,44 & 10,28 & 15,61 & 29,98 & 25,70 & 1,34 & 2,31 & 7,62 & 286,46 & 8119,67 \\
\hline 25,92 & 10,12 & 15,68 & 30,49 & 25,11 & 1,37 & 2,35 & 7,43 & 279,89 & 7673,12 \\
\hline $\mathrm{F}$ & $3,22^{\mathrm{ns}}$ & $1,67^{\mathrm{ns}}$ & $1,66^{\mathrm{ns}}$ & $0,26^{\mathrm{ns}}$ & $2,26^{\mathrm{ns}}$ & $1,10^{\mathrm{ns}}$ & $1,17^{\mathrm{ns}}$ & $2,12^{\mathrm{ns}}$ & $0,62^{\mathrm{ns}}$ \\
\hline $\mathrm{CV}(\%)$ & 4,32 & 3,55 & 5,95 & 5,44 & 5,69 & 3,56 & 5,51 & 4,64 & 9,22 \\
\hline
\end{tabular}


Com base no exposto, ressalta-se a importância da realização de pesquisas que visem aprimorar a tecnologia de aplicação de maturadores em cana-de-açúcar, de maneira a evitar o risco potencial de deriva da pulverização sobre culturas sensíveis. Verificou-se que, no limite de perdas por deriva de até $12 \%$ da dose utilizada de glyphosate como maturador de cana-de-açúcar, a produtividade de grãos não foi afetada, o que garante segurança nas aplicações desse maturador na cultura da cana-de-açúcar localizada nas proximidades de cultivos de milho. Porém, no campo pode-se ocorrer deriva com depósitos de glyphosate em doses maiores ao estudado e refletir em danos à cultura do milho.

Assim, conclui-se que efeitos visuais de fitotoxicidade, alterações nos componentes de crescimento e produção, e ainda redução na produtividade da cultura do milho não são verificados quando ocorre a aplicação de subdoses de até 25,92 $\mathrm{g} \mathrm{ha}^{-1}$ e.a. de glyphosate nos estádios V4 e V8 de desenvolvimento da cultura do milho.

\section{Referências}

Al-Khatib, K.; Claassen, M.M.; Stahlman, P.W.; Geier, P.W.; Regehr, D.L.; Duncan, S.R.; Heer, W.F. Grain sorghum response to simulated drift from glufosinate, glyphosate, imazethapyr, and sethoxydim. Weed Technology, v.17, n.2, p.261-265, 2003.

Anzalone, A.; Arizaleta, M.; Vargas, J.M. Coffee plant (Coffea arabica var. Catuaí response to glyphosate, clomazone, linuron, 2,4D, metsulfuron-methyl, rimsulfuron and clorimurom-ethyl herbicides. Bioagro, v.26, n.1, p.3-12, 2014.

Belz, R.G.; Duke, S.O. Herbicides and plant hormesis. Pest Management Science, v.70, n.5, p.698-707, 2014.

Brasil. Ministério da Agricultura, Pecuária e Abastecimento. Regras para análise de sementes. Brasília: MAPA/ACS, 2009. 399p.
Caputo, M.M.; Beauclair, E.G.F.; Silva, M.A.; Piedade, S.M.S. Resposta de genótipos de canade-açúcar à aplicação de indutores de maturação. Bragantia, v.67, n.1, p.15-23, 2008.

Carvalho, F.P.; Souza, B.P; França, A.C.; Ferreira, E.A.; Franco, M.H.R.; Kasuya, M.C.M.; Ferreira, F.A. Glyphosate drift affects arbuscular mycorrhizal association in coffee. Planta Daninha, v.32, n.4, p.783-789, 2014.

Cedergreen, N. Herbicides can stimulated plant growth. Weed Research, v.48, n.5, p.429-438, 2008.

Correia, N.M.; Villela, G.B. Seletividade de trinexapac-ethyl and sulfometuron-methyl selectivity to young eucalyptus plants. Planta Daninha, v.33, n.2, p.259-266, 2015.

Correia, N.M; Leite, G.J. Selectivity of the plant growth regulators trinexapac-ethyl and sulfometuron-methyl to cultivated species. Scientia Agricola, v.69, n.3, p.194-200, 2012.

Cunha, J.P.A.R. Simulação da deriva de agrotóxicos em diferentes condições de pulverização. Ciência e Agrotecnologia, v.32, n.5, p.1616-1621, 2008.

Dalley, C.D.; Richard Júnior, E.P. Herbicides as ripeners for sugarcane. Weed Science, v.58, n.3, p.329-333, 2010.

Duke, S.O.; Cedergreen, N.; Velini, E.D.; Belz, R.G. Hormesis: Is it an important fator in herbicide use and allelopathy. Outlooks on Pest Management, v.17, n.1, p.29-33, 2006.

EUROPEAN WEED RESEARCH COUNCIL (EWRC). Methods in weeds research. Weed Research, v.4, n.1, p.88, 1964.

Felisberto, P.A.C.; Felisberto, G.; Ramos, A.R.; Timossi, P.C. Sub-doses of 2chloroethylphosphonic acid in vegetative stages of corn cultivation. Revista Brasileira de Herbicidas, v.14, n.2, p.164-170, 2015.

França, A.C.; Carvalho, F.P.; Fialho, C.M.T.; D’Antonino, L.; Silva, A.A.; Santos, J.B.; Ferreira, L.R. Deriva simulada do glyphosate 
em cultivares de café acaiá e catucaí. Planta Daninha, v.31, n.2, p.443-451, 2013.

INMET. Instituto Nacional de Meteorologia. Dados Climatológicos. Disponível em: <http://www.inmet.gov.br/>. Acesso em: 20 jun. 2014.

Köppen, W. Climatologia con un studio de los climas de la Tierra. Buenos Aires, 1931. 320p.

Leite, G.H.; Crusciol, C.A.A.; Silva, M.A. Desenvolvimento e produtividade da cana-deaçúcar após aplicação de reguladores vegetais em meio de safra. Semina: Ciências Agrárias, v.32, n.1, p.129-138, 2011.

Magalhães, P.C.; Silva, J.B.; Durães, F.O.M.; Karam, D.; Ribeiro, L.S. Efeito de doses reduzidas de glyphosate e paraquat simulando deriva na cultura do milho. Planta Daninha, v.19, n.2, p.247-253, 2001.

Means, N.E.; Kremer, R.J. Influence of soil moisture on root colonization of glyphosatetreated soybean by Fusarium species. Communications in Soil Science and Plant Analysis, v.38, n.13-14, p.1713-1720, 2007.

Olesen, C.F.; Cedergreen, N. Glyphosate uncouples gas exchange and chlorophyll fluorescence. Pest Management Science, v.66, n.5, p.536-542, 2010.

Reddy, K.N.; Rimando, A.M.; Duke, S.O.; Nandula, V.K. Aminomethylphosphonic acid accumulation in plant species treated with glyphosate. Journal of Agricultural and Food Chemistry, v.56, n.6, p.2125-2130, 2008.

Schrübbers, L.C.; Valverde, B.E.; Sorensen, J.C.; Cedergreen, N. Glyphosate spray drift in Coffea arabica-Sensitivity of coffee plants and possible use of shikimic acid as a biomarker for glyphosate. Pesticide Biochemistry and Physiology, v.115, p.15-22, 2014.

Silva, M.A.; Gava, G.J.C.; Caputo, M.M.; Sorrilla, P.F.; Bassetto, S.C. Produtividade de colmos e de sacarose em cana-de-açúcar em função da interação entre cultivares e maturadores. Scientia Agraria, v.11, n.4, p.323-329, 2010.

Tuffi Santos, L.D.; Siqueira, C.H.; Barros, N.F.; Ferreira, F.A.; Ferreira, L.R.; Machado, A.F.L. Crescimento e concentração de nutrientes na parte aérea de eucalipto sob efeito de deriva do glyphosate. Cerne, v.13, n.4, p.347-352, 2007.

Velini, E.D.; Alves, E.; Godoy, M.C.; Meschede, D.K.; Souza, R.T.; Duke, S.O. Glyphosate applied at low doses can stimulate plant growth. Pest Management Science, v.64, n. 4, p.489-496, 2008.

Velini, E.D.; Trindade, M.L.B.; Barberis, L.R.M.; Duke, S. O. Growth regulation and other secondary effects of herbicides. Weed Science, v.58, n.3, p.351-354, 2010.

Viana, R.S.; Figueiredo, P.A.M.; Lisboa, L.A.M.; Pascoaloto, I.M. Características morfoanatômicas de folhas de cana-de-açúcar sob efeito residual de maturadores. Revista Brasileira de Herbicidas, v.14, n.4, p.306-314, 2015. 